\title{
Background correction method for DNA microarray image processing
}

\author{
Omar Salem Baans*, Asral Bahari Jambek \\ School of Microelectronic Engineering, Universiti Malaysia Perlis, Arau, 02600 Perlis, Malaysia
}

Received 25th December 2018 / Accepted 25th May 2019

\begin{abstract}
Most microarray image scanning approaches provide an estimation of the intensity of the foreground and background for each spot. Background intensity must be corrected in order to remove the effect of non-specific binding or spatial heterogeneity across the array, but when such corrections are applied many problems appear, such as negative intensity for the spot or high variability of low-intensity log ratios. In this paper, many alternative methods for calculating background intensity are discussed and many approaches for background correction are tested and compared. GenePix, ScanAlyze and QuantArry are the strategies that were reviewed for background locations to extract their intensity. Similarly, to GenePix, a new approach for background calculation was proposed and tested. It shows more accurate results and the occurrences of error become lesser.
\end{abstract}

Keywords: background, correction, DNA, foreground, image-processing, microarray

\section{INTRODUCTION}

Nucleic acid expression regulates the production of proteins which control all cellular processes in the human biological system. The understanding of gene expression and the mechanism of protein production has many applications in terms of diagnosis, staging and finding suitable treatments for diseases. Using the cDNA microarray, it is possible to diagnose rapidly and efficiently the level of gene expression in the sample (Kooperberg et al., 2002; Lee et al., 2000).

There are many commercial and freeware microarray analysis software packages available. Each software program can be separated into three main tasks. The first is gridding or addressing, which is the process of specifying coordinates for every spot on the slide. The second is segmentation, which classifies each pixel as either foreground, corresponding to a spot of interest, or as background, corresponding to error or noise. The third and final task is intensity extraction, which is the step which, for each spot on the array, calculates the green and red foreground fluorescence intensity (Yang et al., 2002; Mabrouk et al., 2013; Ahmad et al., 2014).

The estimation of the background intensity is a very important step to be performed as part of the background correction process. This is because each spot intensity measure includes a contribution to the fluorescence that is not due to hybridization of the mRNA sample to produce spotted DNA. Background intensity can be estimated by more than one method, for example, using the main concentration of pixels located outside the spot mask (Sifakis et al., 2012; Smyth $\&$ Speed, 2003).

The background correction for the spot intensity can usually be performed by subtracting the background intensity from the foreground intensity of the spot, but sometimes negative values appear where the background value is greater than the foreground value for a given spot, which seems illogical. Many studies have

\footnotetext{
*Author for correspondence: Omar Salem Nasser Baans, Division of School of Microelectronic Engineering, Universiti Malaysia Perlis, Arau 02600. Email-omersalim4901@gmail.com
} 
discussed this issue and proposed various methods that can be applied to avoid the problem (Geeleher et al.; Argyropoulos et al., 2010).

The main aim of this paper is to propose and examine a new background correction alternative that can be used to calculate the background value pair (Rb, Gb). In this paper, several microarray background correction methods are discussed. Section A presents the existing algorithm systems for calculating background intensity values, while section $\mathrm{B}$ discusses and compares the different methods used for background correction. Section $\mathrm{C}$ proposes the new background correction in this paper.

\section{A. Most Popular Background Intensity Extraction Methods}

Most microarray analyses define foreground intensity values for red and green fluorescence (Rf, Gf) as the mean or median value of all the pixel values inside the segmented spot mask as shown by the red circle at the center. However, other variations are available for the calculation of the background intensity values for red and green fluorescence $(\mathrm{Rb}, \mathrm{Gb})$ especially with respect to selecting the background region. Among the most popular approaches that utilize the median for the specially-selected region around the spot mask are GenePix, ScanAlyze and QuantArry.

The GenePix package considers the median value of all the pixel values inside the valley region as the foreground intensity value of the spot. The valley region is represented in Figure 1 as four pink rectangular areas surrounding the spot. The background intensity estimation methods implemented in ScanAlyze consider all the pixels that are located outside the spot mask but within the square where the spot lies. This is represented in Figure 1 as the blue square surrounding the red circle. The median of all these pixels is used as the estimated value of the local background intensity of the spot. Finally, the QuantArry method uses the median of every pixel in the area between the two green concentric circles in Figure 1 as the background intensity value for that spot (Fielden et al., 2002; Eisen \& Michael, 2013). While the QuantArry method uses the median of every pixel in the area between the two green concentric circles in Figure 1 as the background intensity value for that spot (Luminomics, 2009; Bengtsson \& Bengtsson, 2006; Affymetrix, 2006).

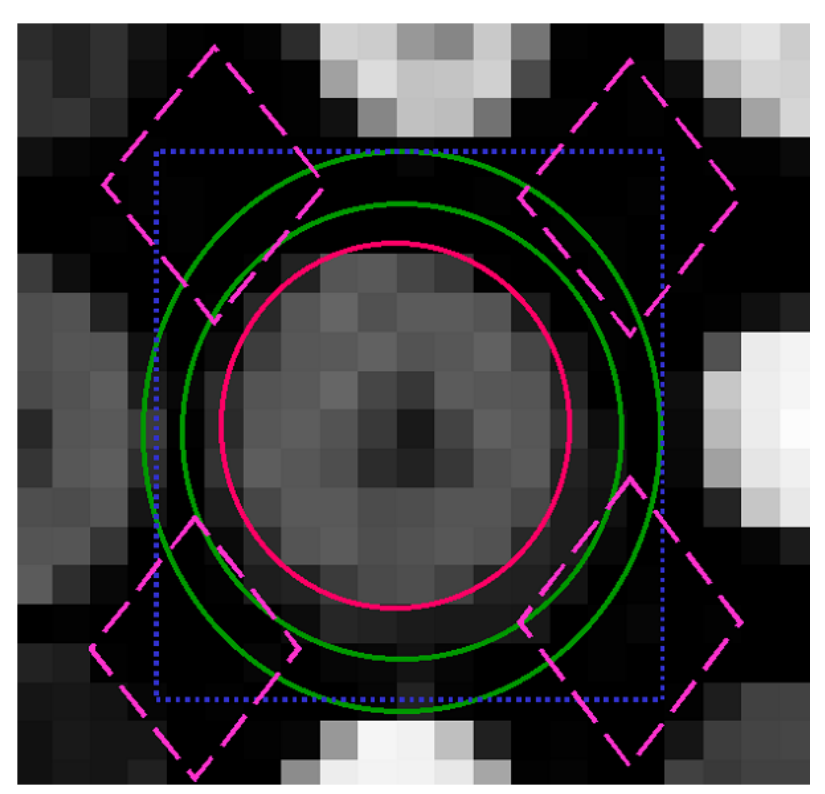

Figure 1. Different background adjustment method.

Table 1. Comparison between different background estimation alternatives.

\begin{tabular}{cccc}
\hline Features & {$[10]$} & {$[11]$} & {$[12]$} \\
\hline Method & GenePix & ScanAlyze & QuantArry \\
\hline Background & $\begin{array}{c}\text { Four } \\
\text { diamonds }\end{array}$ & $\begin{array}{c}\text { Blue } \\
\text { square and } \\
\text { spot mask }\end{array}$ & $\begin{array}{c}\text { Two } \\
\text { concentric } \\
\text { circles }\end{array}$ \\
\hline $\begin{array}{c}\text { Background } \\
\text { calculation }\end{array}$ & Median & Median & Median \\
\hline
\end{tabular}

From the data collected in Table 1, it can be seen that using the median of the pixels' values in the GenePix region decreases the possibility of calculating an erroneous value, because the region is very distant from the spot and will not include any pixel that belongs to the spot. Thus, miscalculation is avoided for the bright pixel. However, estimation inaccuracy may occur in the other approaches, e.g., in ScanAlyze where there is overlap between the foreground and background areas, and hence it is inevitable that the values of pixels belonging to the spot will be included in the calculation.

\section{B. Background Correction Method}

The usual calculation method for finding the corrected value for the intensity of the spot for a two-color microarray is that of subtracting the values of the background intensity of the two colors, red and green ( $\mathrm{Rb}, \mathrm{Gb})$ from the values of the foreground intensity of the two colors (Rf, Gf). This allows the true spot intensity $(R, G)$ to 
be more accurately evaluated. This is because the observed foreground intensity value is the sum of the background value and the true intensity value of the spot (Ritchie et al., 2007; Yin et al., 2005).

A simpler method for avoiding negative intensity values was presented by Edwards using a local median of the background values. This method is similar to the standard method, but when the value after subtraction is less than a specific small threshold value, it undergoes a monotonic function. Whereas when it is larger than the threshold value, it can be considered to be the true intensity of the spot (Edwards, 2003; Yang et al., 2002).

\section{Proposed Method}

From the algorithms in section II, the criteria of DNA microarray image processing can be improved by proposing a new algorithm specially in calculating background correction. It was mentioned before that the best method for background location is GenePix. However, to get more accurate result, the number of background square locations should be more than four to take more locations especially on the four edges of each side as well the red rectangular in Figure 2.

\section{MATERIALS AND METHOD}

As it is mentioned before in this article, to extract the intensity form DNA microarray slide, the user must go through three main process: addressing, segmentation, and intensity extraction. Using Matlab R2016b, we developed a code that can extract the intensity for 100 spots automatically. As it can be seen in the flow chart in Figure 3, matlab code starts by importing the microarray image slide as the image in Figure 4. Then, it precedes gridding process by convert the image to grayscale image, estimate spot spacing by autocorrelation, locate the centers of spots, draw gridding lines to separate each spot. After that, it proceeds by segmentation using logically combine local and global thresholds.

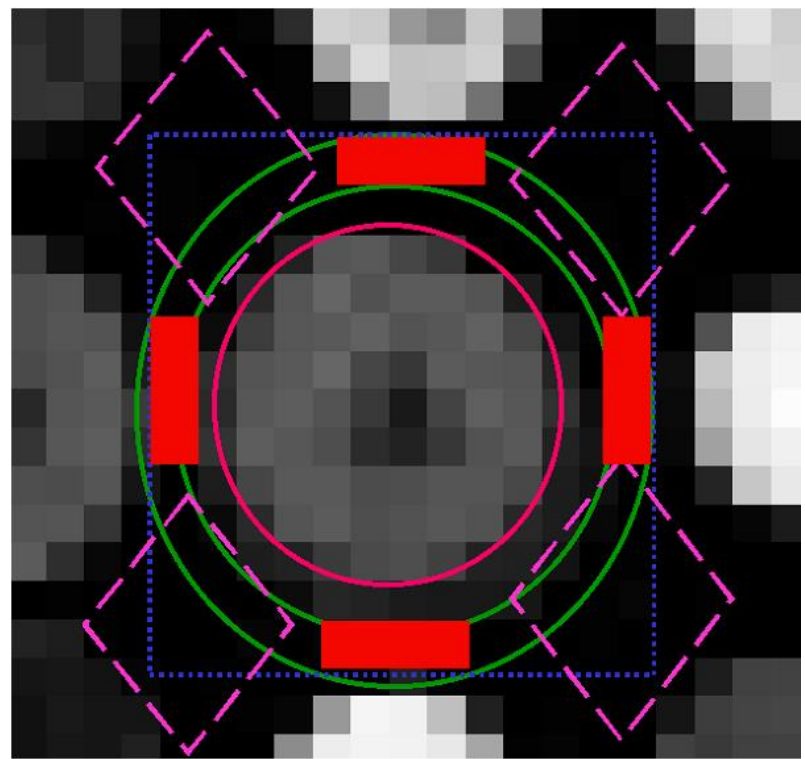

Figure 2. Background proposed locations.

Background intensity is calculated by taking the median of the four squares in the corner of each spot. Similarly, we tested adding another four rectangular on the middle edge of each side of each spot. Background correction performed using Edward algorithm (Edwards, 2003). Finally, the intensity of each spot was extracted by taking the median of the spot mask.

The mathematical Equations of background corrections starts firstly by calculating the red and green foreground (Rf, Gf) by finding the median value of the pixels inside each spot mask and calculate red and green background intensities $(\mathrm{Rb}, \mathrm{Gb})$ by finding the median value of the pixels inside the local background areas of the spot. Then, the spot intensity was calculated by subtracting the background from the foreground as in Equation (1) and (2):

$$
\begin{aligned}
& R=R f-R b \\
& G=G f-G b
\end{aligned}
$$




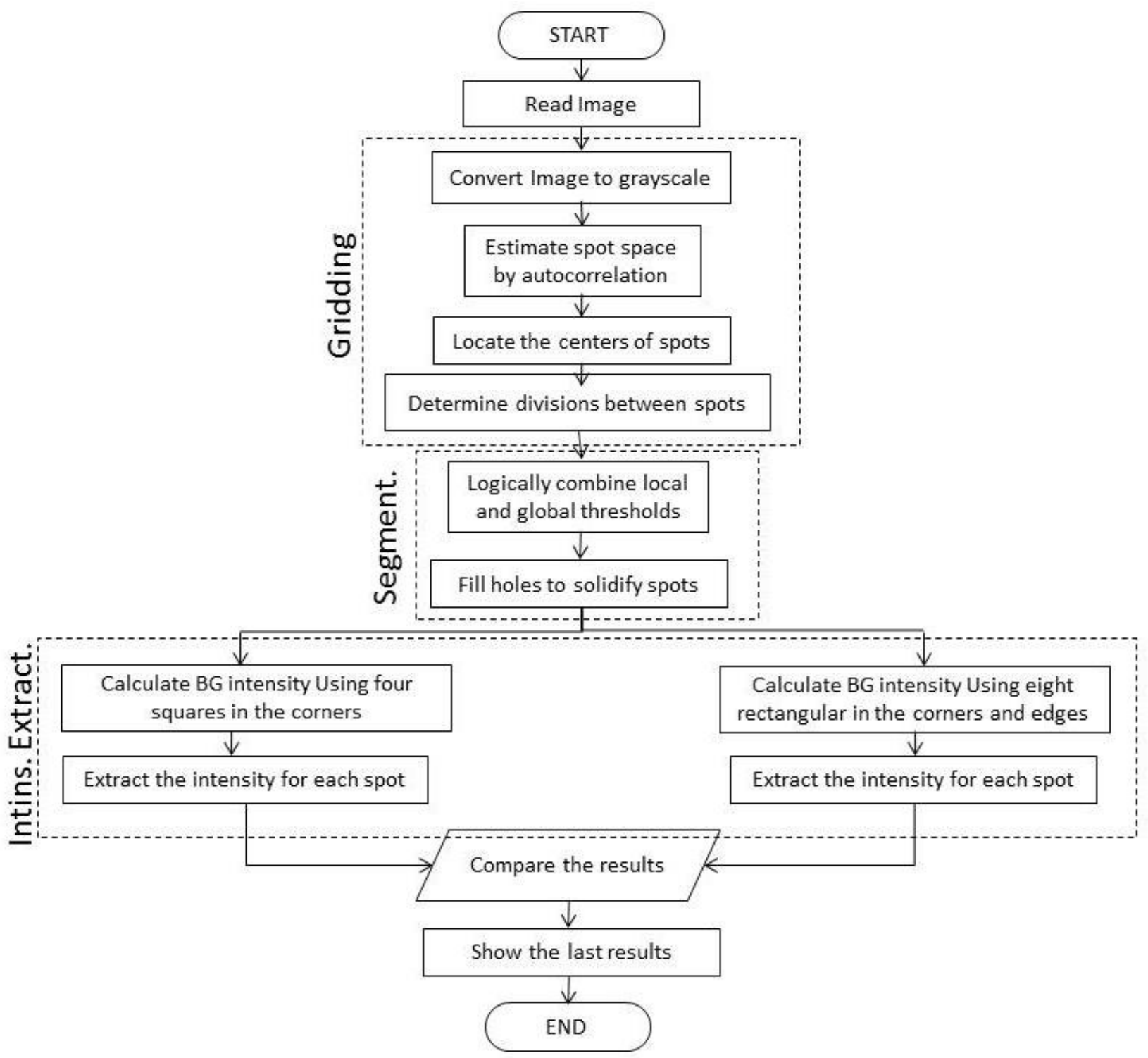

Figure 3. Methodology flow chart.

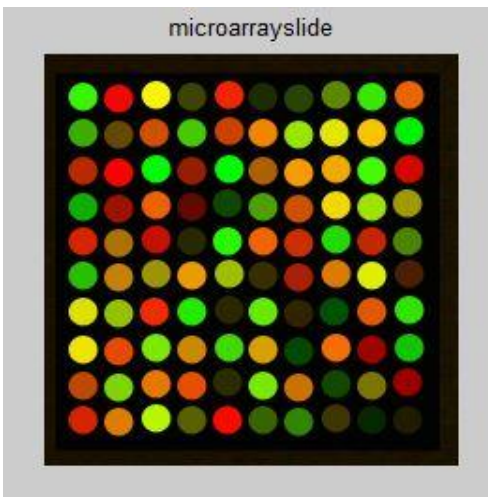

Figure 4. Microarray ideal background image slide.

The results of GenePix and this new algorithm compared and discussed. Another 6 more images such as image in Figure 5 compiled using this code with different background values while the foreground is similar. Thus, the conclusion becomes easier to pick the most accurate method.

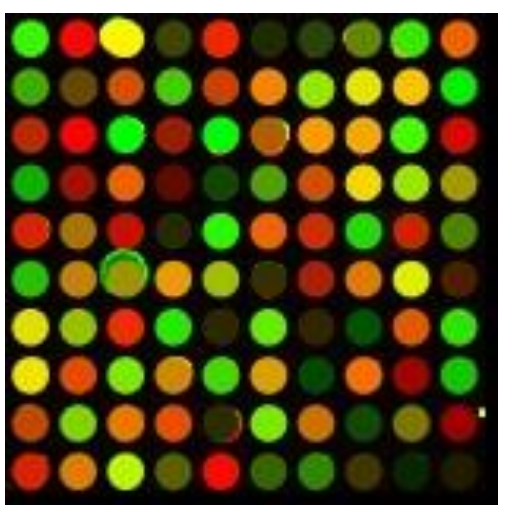

Figure 5. Microarray image slide. 
In order to validate the results, another three microarray images as in Figure 6, each image consists of one hundred spots. These images utilized in this work are real pictures obtained from a public database of the Princeton University microarray database. Princeton University microarray database provide the measured intensity information for each image (Ball et al., 2005). Thus, this information was used as a reference to compare and validate this research results according to four parameters, these parameters are PSNR (Peak Signal to Noise Ratio).
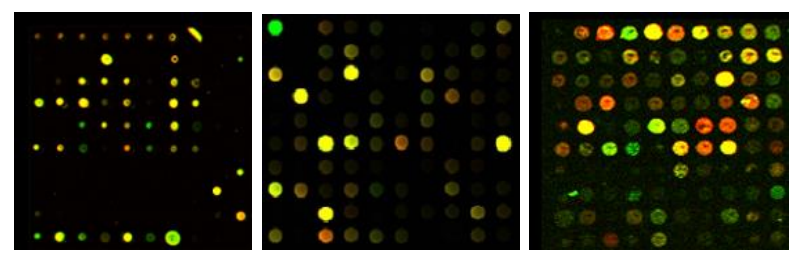

Figure 6. Princeton DNA microarray images to validate the results.

\section{RESULTS AND DISCUSSION}

First of all, almost all the methods share a similarity especially when we compare the difference between the green and the red intensity for the same spots. The different usually fluctuating between zeros to 6 pixels. Matrix 1 below shows the results for Ideal Microarray Image that has a similar background values by 3 pixels and that's why once we tried to show 100\% similarities between GenePix BG location and the proposed method.

Matrix 2 shows the intensity extraction for DNA microarray image in Figure 5. Therefore, from matrix 1 and 2 the reader can know the background intensity for each spot in Figure 5 because it is fix number equals to three for every spot in Figure 4. Thus, red background intensity in the first in Figure 5 is equal to 2. However, green background intensity for the same spot is equal to 18 and so on. From this we can see how important of performing background correction before proceeding to extract the intensity of the spot.

\begin{tabular}{|c|c|c|c|c|c|c|c|c|c|}
\hline \multicolumn{10}{|c|}{ Red_Intensity $=$} \\
\hline 51 & 243 & 251 & 58 & 242 & 25 & 37 & 92 & 51 & 234 \\
\hline 58 & 100 & 208 & 68 & 202 & 241 & 149 & 228 & 246 & 0 \\
\hline 185 & 251 & 0 & 150 & 0 & 173 & 247 & 239 & 65 & 211 \\
\hline 11 & 156 & 235 & 96 & 14 & 74 & 204 & 240 & 154 & 158 \\
\hline 215 & 171 & 195 & 37 & 37 & 239 & 201 & 29 & 198 & 75 \\
\hline 35 & 193 & 156 & 234 & 156 & 54 & 169 & 219 & 219 & 72 \\
\hline 220 & 147 & 239 & 33 & 40 & 96 & 47 & 0 & 222 & 44 \\
\hline 237 & 229 & 122 & 203 & 64 & 213 & 0 & 244 & 156 & 16 \\
\hline 191 & 124 & 231 & 230 & 42 & 118 & 202 & 15 & 121 & 151 \\
\hline 215 & 221 & 181 & 86 & 250 & 51 & 47 & 61 & 5 & 29 \\
\hline \multicolumn{10}{|c|}{ Green_Intensity = } \\
\hline 245 & 3 & 242 & 66 & 33 & 41 & 66 & 133 & 231 & 97 \\
\hline 171 & 70 & 77 & 200 & 62 & 130 & 227 & 226 & 193 & 238 \\
\hline 37 & 0 & 252 & 27 & 252 & 93 & 152 & 167 & 249 & 4 \\
\hline 173 & 15 & 95 & 6 & 67 & 158 & 78 & 213 & 225 & 151 \\
\hline 32 & 112 & 13 & 38 & 246 & 97 & 43 & 216 & 34 & 129 \\
\hline 190 & 127 & 145 & 152 & 189 & 43 & 28 & 119 & 236 & 28 \\
\hline 221 & 191 & 41 & 231 & 37 & 233 & 33 & 79 & 86 & 225 \\
\hline 225 & 71 & 231 & 134 & 214 & 158 & 69 & 107 & 0 & 194 \\
\hline 69 & 211 & 116 & 75 & 41 & 231 & 111 & 69 & 115 & 0 \\
\hline 32 & 122 & 244 & 94 & 5 & 98 & 131 & 51 & 36 & 25 \\
\hline
\end{tabular}

Matrix 1. Results of background correction method for ideal image.

\begin{tabular}{cccccccccc}
\hline \multicolumn{2}{c}{ Red_Intensity $=$} & & & & & & & \\
52 & 214 & 239 & 50 & 218 & 22 & 33 & 84 & 50 & 217 \\
55 & 89 & 186 & 67 & 184 & 222 & 144 & 216 & 230 & 3 \\
167 & 224 & 0 & 134 & 0 & 158 & 225 & 222 & 66 & 189 \\
10 & 138 & 214 & 82 & 12 & 70 & 186 & 225 & 150 & 147 \\
192 & 156 & 169 & 30 & 36 & 219 & 183 & 24 & 173 & 70 \\
36 & 177 & 112 & 217 & 149 & 45 & 151 & 197 & 209 & 61 \\
208 & 141 & 212 & 37 & 34 & 95 & 37 & 0 & 201 & 45 \\
225 & 203 & 118 & 188 & 60 & 201 & 0 & 228 & 139 & 15 \\
173 & 121 & 209 & 204 & 33 & 113 & 181 & 12 & 110 & 133 \\
194 & 203 & 177 & 78 & 222 & 49 & 47 & 52 & 5 & 21
\end{tabular}

\begin{tabular}{cccccccccc}
\multicolumn{2}{c}{ Green_Intensity $=$} \\
230 & 7 & 234 & 61 & 36 & 38 & 61 & 126 & 215 & 96 \\
163 & 68 & 77 & 189 & 62 & 129 & 216 & 218 & 188 & 221 \\
38 & 2 & 236 & 27 & 235 & 92 & 149 & 163 & 234 & 8 \\
161 & 19 & 96 & 7 & 61 & 151 & 78 & 207 & 213 & 146 \\
34 & 110 & 15 & 34 & 230 & 97 & 45 & 194 & 33 & 122 \\
180 & 122 & 109 & 148 & 181 & 41 & 29 & 116 & 228 & 27 \\
214 & 181 & 42 & 214 & 33 & 220 & 31 & 72 & 87 & 212 \\
219 & 72 & 220 & 131 & 203 & 153 & 62 & 106 & 3 & 181 \\
69 & 201 & 113 & 75 & 38 & 220 & 110 & 63 & 110 & 1 \\
36 & 121 & 235 & 89 & 11 & 91 & 123 & 47 & 31 & 22 \\
\hline
\end{tabular}

Matrix 2. Results intensity extraction for DNA microarray image in Figure 5.

Matrix 3 shows the different between using eight rectangular instead of four to calculate background intensity. As it can be seen below, there is for most of the spots and these different is varies between 0 and 6 . However, one of the green spots shows a different of 32 pixels. That because when the user increases the locations of 
background the accuracy will be increased as well and the occurrences of the error will be decreased.

\begin{tabular}{|c|c|c|c|c|c|c|c|c|c|}
\hline \multicolumn{10}{|c|}{ R_bg_diff $=$} \\
\hline 2 & -2 & -3 & 0 & -4 & -1 & -1 & -1 & 0 & -2 \\
\hline 0 & -3 & -3 & 1 & -1 & -3 & -1 & -2 & -2 & 2 \\
\hline-2 & -6 & 0 & -4 & 0 & -3 & -6 & -3 & 1 & -3 \\
\hline 0 & -4 & -4 & -4 & 0 & -1 & -6 & -2 & 0 & -1 \\
\hline-6 & -4 & -3 & 1 & 3 & -3 & -2 & 1 & -4 & 1 \\
\hline 1 & -4 & -4 & -1 & 0 & -3 & -3 & -5 & 0 & -1 \\
\hline 0 & 2 & -3 & 5 & 1 & 2 & 0 & 0 & -3 & 3 \\
\hline-2 & -3 & 2 & -2 & 2 & -3 & 0 & -4 & -3 & 2 \\
\hline-2 & 3 & -3 & -4 & -3 & 1 & -4 & 0 & -2 & -3 \\
\hline-3 & -2 & 1 & -1 & -2 & 1 & 3 & 0 & 1 & 0 \\
\hline \multicolumn{10}{|c|}{ G_bg_diff $=$} \\
\hline-3 & 0 & -3 & -1 & 1 & -1 & 0 & -1 & -2 & 0 \\
\hline-2 & 0 & 1 & -2 & 0 & -1 & -3 & -1 & -2 & -3 \\
\hline 1 & 2 & -2 & 0 & -2 & 0 & 0 & -1 & -3 & 1 \\
\hline 0 & 2 & 1 & 2 & 0 & 0 & 1 & -1 & -4 & 0 \\
\hline 1 & 0 & 0 & -1 & -3 & 1 & 1 & -2 & 2 & -1 \\
\hline-1 & -1 & -32 & -1 & -1 & 1 & 2 & 0 & 0 & 0 \\
\hline-1 & -3 & 2 & -2 & -1 & -2 & -1 & -2 & 3 & -2 \\
\hline 1 & 3 & 0 & 0 & -2 & 0 & -1 & 2 & 1 & -2 \\
\hline 1 & -2 & 1 & 1 & 0 & -1 & 1 & -2 & 0 & 1 \\
\hline 3 & 0 & -1 & -1 & 4 & -1 & -2 & -1 & 0 & -1 \\
\hline
\end{tabular}

Matrix 3. The different between using eight rectangular instead of four to calculate background intensity.

Table 2 compares background corrections method applied on three DNA microarray images in Figure 6. The results of GenePix and the proposed method were compared with the Princeton database as a reference. The comparison was done depending the Peak Signal to Nois Ration PSNR as parameters for the comparison.

Table 2. Background correction comparisons between the proposed method and GenePix method.

\begin{tabular}{cccc}
\hline Method & $\begin{array}{c}\text { Image } \\
\text { (1) }\end{array}$ & $\begin{array}{c}\text { Image } \\
\text { (2) }\end{array}$ & $\begin{array}{c}\text { Image } \\
\text { (3) }\end{array}$ \\
\hline GenePix & $40.22 \mathrm{~dB}$ & $43.17 \mathrm{~dB}$ & $41.39 \mathrm{~dB}$ \\
Proposed & $40.35 \mathrm{~dB}$ & $43.18 \mathrm{~dB}$ & $41.47 \mathrm{~dB}$ \\
\hline
\end{tabular}

According to Table 2, the accuracy of the proposed method is much better than GenePix methods where PSNR for the proposed is greater than PSNR for GenePix method for all the tested three images.

\section{CONCLUSION}

In this paper, a number of background correction calculations and algorithms were reviewed. The differences and similarities of the existing systems were studied in order to identify criteria for selecting the best program, i.e., the program which gives results closest to the true intensity values. Three methods for allocating and calculating the background intensity values were discussed and compared. These methods were GenePix, ScanAlyze and QuantArry. To correct the background intensity, a new algorithm was applied on microarray slide image using MATLAB in order to find the most accurate intensity value of the two-color microarray for each spot; and the result for that method and other existing methods were calculated and compared. Based on the findings, it is sufficed to state that choosing more background locations in the corners and the sides is the best way to find the most accurate background intensity.

\section{ACKNOWLEDGMENTS}

This research was funded by ScienceFund, Ministry of Science, Technology and Innovation (MOSTI), Malaysia (2015).

\section{REFERENCES}

Affymetrix Expression Console Software Version 1.0. 2006. User Guide. Santa Clara: Affymetrix Inc.

Ahmad, M. M., Jambek, A. B., \& Mashor, M. Y. 2014. August. A study on microarray image gridding techniques for DNA analysis. In 2014 2nd International Conference on Electronic Design (ICED) (pp. 171-175). IEEE.

Argyropoulos, C., Daskalakis, A., Nikiforidis, G. C., \& Sakellaropoulos, G. C. 2010. Background adjustment of cDNA microarray images by Maximum Entropy distributions. Journal of Biomedical Informatics 43(4): 496-509.

Ball, C. A., Awad, I. A., Demeter, J., Gollub, J., Hebert, J. M., Hernandez-Boussard, T., Jin, H., Matese, J. C., Nitzberg, M., Wymore, F., \& Zachariah, Z. K. 2005. The Stanford Microarray Database accommodates additional microarray platforms and data formats. Nucleic Acids Research 33(suppl_1): D580-D582. 
Bengtsson, A. \& Bengtsson, H. 2006. Microarray image analysis: background estimation using quantile and morphological filters. BMC Bioinformatics 7(1): 96.

Edwards, D. 2003. Non-linear normalization and background correction in one-channel cDNA microarray studies. Bioinformatics 19(7): 825-833.

Eisen, M. 2013. "ScanAlyze user manual." Standford Univ. US.

Fielden, M. R., Halgren, R. G., Dere, E., \& Zacharewski, T. R. 2002. GP3: GenePix post-processing program for automated analysis of raw microarray data. Bioinformatics 18(5): 771-773.

Geeleher, M. P., Morris, D., Golden, A., \& Hinde, J. BioconductorBuntu Users Manual.

Kooperberg, C., Fazzio, T. G., Delrow, J. J., \& Tsukiyama, T. 2002. Improved background correction for spotted DNA microarrays. Journal of Computational Biology 9(1): 55-66.

Lee, M. L. T., Kuo, F. C., Whitmore, G. A., \& Sklar, J. 2000. Importance of replication in microarray gene expression studies: statistical methods and evidence from repetitive cDNA hybridizations. Proceedings of the National Academy of Sciences 97(18): 9834-9839.

Luminomics, G. S. I. 2009. "QuantArray Analysis Software, Operator's Manual." GSI Luminomics.

Mabrouk, M., Fouad, I., \& Sharawy, A. 2013. Automated statistical and morphological based gridding methods for noisy microarray image processing. Journal of Bioinformatics and Intelligent Control 2(4): 265-275.

Ritchie, M. E., Silver, J., Oshlack, A., Holmes, M., Diyagama, D., Holloway, A., \& Smyth, G. K., 2007. A comparison of background correction methods for two-colour microarrays. Bioinformatics 23(20): 2700-2707.

Sifakis, E. G., Prentza, A., Koutsouris, D., \& Chatziioannou, A. A. 2012. Evaluating the effect of various background correction methods regarding noise reduction, in twochannel microarray data. Computers in Biology and Medicine 42(1): 19-29.

Smyth, G. K. \& Speed, T. 2003. Normalization of cDNA microarray data. Methods 31(4): 265-273.

Yang, Y. H., Buckley, M. J., Dudoit, S., \& Speed, T. P. 2002. Comparison of methods for image analysis on cDNA microarray data. Journal of Computational and Graphical Statistics 11(1): 108-136.

Yin, W., Chen, T., Zhou, S. X., \& Chakraborty, A., 2005. Background correction for $\mathrm{cDNA}$ microarray images using the TV+ L 1 model. Bioinformatics 21(10): 2410-2416. 